\title{
Condition of Optical Systems Independent of Frequency for Wide Field-of-View Radio Telescopes
}

\author{
Hiroaki Imada, Makoto Nagai, Masaru Kino, Masumichi Seta, Shun Ishii, and Naomasa Nakai
}

\begin{abstract}
We present a condition of optical systems to form images independent of frequency. The condition independent of frequency has been known in case of a single Gaussian beam on an optical axis, but has not been known in case of a beam propagated off the axis or tilted beam, which appears in a wide field-of-view telescope. We first show relations between an arbitrary electric field on an object plane and an induced one on an image plane after passing through a lens with calculating Fresnel diffraction integrals. If the lens formula is met, there is a one-to-one correspondence between points on the object and image plane. This result enables to use geometrical optical methods. The condition and relations derived here were confirmed by simulations. We also applied them to a wide fieldof-view telescope.
\end{abstract}

Index Terms-Electromagnetic diffraction.

\section{INTRODUCTION}

W IDE field-of-view (FOV) telescopes for observing in $\mathrm{mm}$ or sub- $\mathrm{mm} / \mathrm{THz}$ regions have been developed, e.g., SPT [1], ACT [2], and are being developed, e.g., CCAT [3]. In the optical and infrared regions, telescopes with FOV of more than 1 degree, e.g., Schmidt telescopes, were developed, or designed using geometrical optics, e.g., LSST [4].

There are a few characteristics of a wide field of view. Firstly, in general, wide FOV systems have many mirrors to cancel aberrations, not only to transmit beams. Secondly, we can now use a free-form surface, e.g., [5], which allows us to have more choice in designing optical systems. Free-form surfaces include not only conic surfaces but also higher order polynomial surfaces. Transformation of a spherical wavefront into another spherical one by a conic surface is traditional and is a special case of general one such as transformation of an aspherical wavefront into spherical one. Finally, beams propagate various paths, i.e., most of them are kept away from and tilted against the optical axis. It means that each beam reflects (passes through) a part of mirrors (lenses) corresponding to the beam and that a concept of "pupil" grows greatly important.

We now focus on the influence of diffraction seen in a wide FOV system and field distributions which depend on frequencies generally. It has not been studied sufficiently in both radio and optical regions. In case of the Gaussian beam, it is well known that the beam radius grows with beam

H. Imada, M. Nagai, M. Seta, and N. Nakai are with University of Tsukuba, Tsukuba, Ibaraki 305-8571 Japan (corresponding author to provide phone: +81-29-853-5600 (ext.8299); e-mail: s1330093@u.tsukuba.ac.jp).

M. Kino is with Kyoto University, Kyoto, Kyoto 606-8502 Japan.

S. Ishii was with University of Tsukuba. He is now with the University of Tokyo, Mitaka, Tokyo 181-0015 Japan.

Manuscript received; revised. propagation as a function of frequency. It was shown in [6], for Gaussian beams propagating on an optical axis, that the beam radius at specific positions does not depend on frequency. The conditions showed in [6] are not sufficient when we apply them to a wide FOV system due to the wide FOV characteristics. We have to find applicable conditions of a wide FOV system.

Reference [7] calculated an electric field induced by an arbitrary electric field expanded into a summation of fundamental and higher order mode Gaussian-Hermite beams. Using beammode transfer matrices, [7] derived the relations of the Fourier Optics and showed that the distribution on the image plane can be calculated by scaling or magnifying that on the object plane independently of frequency in case that the two planes met the lens formula. There, however, seems to be mathematically unclear points in deriving the relations; [7] assumed the lens formula and discussed frequency-independent images with an analogy between a Fourier Optics system consisting of two transformers and a single lens system in spite of each system having different forms of beam-mode transfer matrices.

We can use the Fresnel diffraction integral instead of using beam-mode transfer matrices. Reference [8] showed the calculation of the Fresnel diffraction integral but did not integrate on the object plane or take into consideration finally quadratic terms present in the phase terms.

We would like conditions, relations or equations to form images independent of frequencies applicable to a wide FOV system as simply as possible, i.e., without applying the condition of [6] as many times as the number of mirrors. We would also like to show a derivation more clearly in a mathematical points of view. We show in this paper that an electric field on the image plane is calculated by the Fresnel diffraction integral on a single lens and the object plane, and investigate relations of field distributions and of wavefront between the object and image plane in Section II. Relations between the Geometrical optics and the derivation in Section II are treated briefly in Section III. Numerical simulations verify the condition and equation derived here in Section IV and show in Section V that we can apply them to a wide FOV telescope.

\section{Calculating Fresnel Diffraction Integral}

Fig. 1 represents the model for calculating the Fresnel diffraction integral. The coordinate is set as shown in Fig. 1 and an electromagnetic wave with a wavenumber of $k$ propagates from the negative region of $z$ to the positive. There is an axially symmetric lens at $z=0$ with a radius of $R$, a focal length of $f>0$. We assume for simplicity that the lens has no aberrations, though effects of aberrations appear in higher 


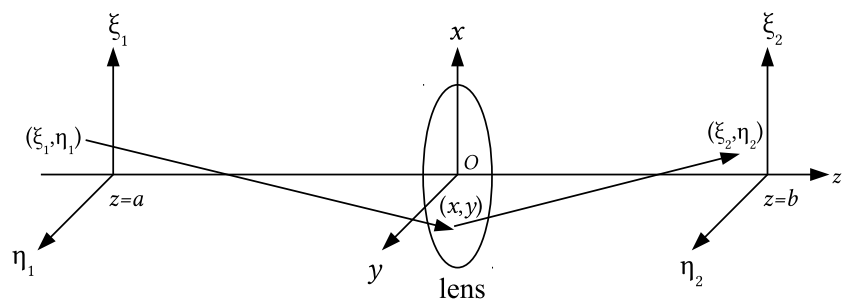

Fig. 1. Model for calculating the Fresnel diffraction integral. There is a lens with a focal length of $f$ at $z=0$. The coordinates $\left(\xi_{1}, \eta_{1}\right)$ and $\left(\xi_{2}, \eta_{2}\right)$ are defined as shown. This figure is in case of $a<0$ and $b>0$, while our discussion goes in parallel no matter what their signs are.

oder terms of wavefront transformation than we take here into consideration. We refer to the plane at $z=a$ as an object plane and to the plane at $z=b$ as an image plane, respectively. Coordinates $\left(\xi_{1}, \eta_{1}\right)$ and $\left(\xi_{2}, \eta_{2}\right)$ are on the object and image plane, respectively. $E_{\text {obj }}\left(\xi_{1}, \eta_{1}\right), E_{\operatorname{lns}}(x, y)$, and $E_{\text {img }}\left(\xi_{2}, \eta_{2}\right)$ are fields expressed by a complex number on the object plane, lens, and image plane, respectively. The time dependence is assumed as $\exp (-i \omega t)$, where $i=\sqrt{-1}, \omega=c k$, and $c$ is the speed of light.

We assume the paraxial approximation that $1 / k \ll$ $x, y, \xi, \eta, R \ll a, b, f . R$ is also assumed to be large enough to carry high spatial frequencies. Paying attention to the phase transformation at the lens, and using the Fresnel diffraction integral, we can express the fields as follows;

$$
\begin{aligned}
E_{\operatorname{lns}}(x, y) & =\frac{i k}{2 \pi a} \exp \left(-i k a-i k \frac{x^{2}+y^{2}}{2 a}\right) \\
& \times \int \mathrm{d} \xi_{1} \int \mathrm{d} \eta_{1} E_{\mathrm{obj}}\left(\xi_{1}, \eta_{1}\right) \\
& \times \exp \left(i k \frac{x \xi_{1}+y \eta_{1}}{a}-i k \frac{\xi_{1}^{2}+\eta_{1}^{2}}{2 a}\right) \\
E_{\mathrm{img}}\left(\xi_{2}, \eta_{2}\right) & =-\frac{i k}{2 \pi b} \exp \left(i k b+i k \frac{\xi_{2}^{2}+\eta_{2}^{2}}{2 b}\right) \\
& \times \int \mathrm{d} x \int \mathrm{d} y E_{\operatorname{lns}}(x, y) \exp \left(-i k \frac{x^{2}+y^{2}}{2 f}\right. \\
& \left.\quad-i k \frac{x \xi_{2}+y \eta_{2}}{b}+i k \frac{x^{2}+y^{2}}{2 b}\right) .
\end{aligned}
$$

Substituting (1) for (2) and re-arranging the integrals, we relate the fields on the object and image plane:

$$
\begin{aligned}
E_{\text {img }}\left(\xi_{2}, \eta_{2}\right) & =\frac{k^{2}}{4 \pi^{2} a b} \exp \left(i k(b-a)+i k \frac{\xi_{2}^{2}+\eta_{2}^{2}}{2 b}\right) \\
& \times \int \mathrm{d} \xi_{1} \int \mathrm{d} \eta_{1} E_{\text {obj }}\left(\xi_{1}, \eta_{1}\right) \exp \left(-i k \frac{\xi_{1}^{2}+\eta_{1}^{2}}{2 a}\right) \\
& \times \int \mathrm{d} x \int \mathrm{d} y \exp \left[-i k \frac{x^{2}+y^{2}}{2}\left(\frac{1}{f}+\frac{1}{a}-\frac{1}{b}\right)\right. \\
& \left.+i k x\left(\frac{\xi_{1}}{a}-\frac{\xi_{2}}{b}\right)+i k y\left(\frac{\eta_{1}}{a}-\frac{\eta_{2}}{b}\right)\right] . \text { (3) }
\end{aligned}
$$

Equation (3) shows that the distribution $E_{\mathrm{img}}\left(\xi_{2}, \eta_{2}\right)$ on the image plane depends on the field distribution $E_{\text {obj }}\left(\xi_{1}, \eta_{1}\right)$ on the object plane and the wavenumber $k$. We seek conditions for which an amplitude of the distribution $E_{\mathrm{img}}\left(\xi_{2}, \eta_{2}\right)$ except for a phase term becomes independent of the wavenumber $k$, that is,

$$
E_{\text {img }}\left(\xi_{2}, \eta_{2}\right)=C(a, b) E_{\text {obj }}\left(\xi_{1}, \eta_{1}\right) \exp (i k \Delta),
$$

where $C(a, b)$ is a real coefficient and $\Delta$ is an amount representing how a phase advances or retards. Equation (4) is a special case in terms of being independent of the wavenumber as a result of superposition of the diffracted wave.

To evaluate the integrals, we change notation of the variables and parameters below;

$$
\begin{aligned}
A & :=\frac{1}{f}+\frac{1}{a}-\frac{1}{b}, \\
\rho \cos \varphi & :=\frac{\xi_{1}}{a}-\frac{\xi_{2}}{b} \\
\rho \sin \varphi & :=\frac{\eta_{1}}{a}-\frac{\eta_{2}}{b}, \\
r \cos \theta & :=x \\
r \sin \theta & :=y .
\end{aligned}
$$

We define the integral of $x, y$ as $I$ and integrate over $\theta$

$$
\begin{array}{rl}
I:=\int \mathrm{d} & x \int \mathrm{d} y \exp \left[-i k A \frac{x^{2}+y^{2}}{2}\right. \\
& \left.+i k x\left(\frac{\xi_{1}}{a}-\frac{\xi_{2}}{b}\right)+i k y\left(\frac{\eta_{1}}{a}-\frac{\eta_{2}}{b}\right)\right] \\
= & 2 \pi \int_{0}^{R} \mathrm{~d} r \exp \left(-i k A \frac{r^{2}}{2}\right) J_{0}(k r \rho),
\end{array}
$$

where $J_{0}$ is the zero order Bessel function of the first kind. Expanding $J_{0}$, we obtain

$$
\begin{aligned}
I= & 2 \pi \sum_{m=0}^{\infty} \frac{(-1)^{m} k^{2 m} \rho^{2 m}}{(m !)^{2} 2^{2 m}} \\
& \times \int_{0}^{R} \mathrm{~d} r r^{2 m+1} \exp \left(-i k A \frac{r^{2}}{2}\right) .
\end{aligned}
$$

The integral of $r$ in (7) contains higher order terms of $k$ due to the exponential and becomes a coefficient of (3) without integrated by $\rho$ and $\varphi$. We then require

$$
A=\frac{1}{f}+\frac{1}{a}-\frac{1}{b}=0
$$

to obtain a relation in the form of (4). Equation (8) represents the well-known lens formula. Images independent of frequency can be obtained where and only where this formula holds. Note that we can expect $\rho$ to be nearly zero because there are terms including the higher orders of $k$. Substituting (8) for the integral $I$, we obtain

$$
I=2 \pi R^{2} \int_{0}^{1} \mathrm{~d}\left(\frac{r}{R}\right) \frac{r}{R} J_{0}(k r \rho / R)=\pi R^{2} \frac{2 J_{1}(k R \rho)}{k R \rho},
$$


and can write the distribution as follows;

$$
\begin{aligned}
& E_{\text {img }}\left(\xi_{2}, \eta_{2}\right)=\frac{R^{2} k^{2} a}{4 \pi b} \exp \left(i k(b-a)+i k \frac{\xi_{2}{ }^{2}+\eta_{2}{ }^{2}}{2 b}\right) \\
& \times \int \mathrm{d} \rho \int \mathrm{d} \varphi \rho E_{\text {obj }}\left(a \rho \cos \varphi+\frac{a \xi_{2}}{b}, a \rho \sin \varphi+\frac{a \eta_{2}}{b}\right) \\
& \times \exp \left(-i k \frac{\left(a \rho \cos \varphi+a \xi_{2} / b\right)^{2}+\left(a \rho \sin \varphi+a \eta_{2} / b\right)^{2}}{2 a}\right) \\
& \times \frac{2 J_{1}(k R \rho)}{k R \rho} .
\end{aligned}
$$

Equation (10) allows us to adopt an arbitrary field distribution as the distribution $E_{\mathrm{obj}}$ as far as it exists physically, i.e., it is a constant or decreases to zero at infinity, because $2 J_{1}(x) / x$ decreases rapidly as $x \gg 1$ where it behaves like $2 \sqrt{2 / \pi x^{3}} \cos (x-3 \pi / 4)$ asymptotically. In addition, the field $E_{\mathrm{img}}\left(\xi_{2}, \eta_{2}\right)$ is determined by only the field at a point $\left(a \xi_{2} / b, a \eta_{2} / b\right)$ because the paraxial approximation makes $k R$ greater than unity. In other words, the distribution $E_{\text {obj }}$ can contribute only when $\rho$ is almost zero. Using (8), we obtain

$$
\begin{aligned}
& E_{\text {img }}\left(\xi_{2}, \eta_{2}\right)=\frac{R^{2} k^{2} a}{4 \pi b} \exp \left(i k(b-a)-i k \frac{a \xi_{2}^{2}+\eta_{2}^{2}}{b f}\right) \\
& \times \int \mathrm{d} \rho \int \mathrm{d} \varphi \rho E_{\mathrm{obj}}\left(a \rho \cos \varphi+\frac{a \xi_{2}}{b}, a \rho \sin \varphi+\frac{a \eta_{2}}{b}\right) \\
& \times \exp \left[-i \frac{k a \rho}{2}\left(\rho+\frac{2\left(\xi_{2} \cos \varphi+\eta_{2} \sin \varphi\right)}{b}\right)\right] \\
& \times \frac{2 J_{1}(k R \rho)}{k R \rho} .
\end{aligned}
$$

We now consider the integral of the Bessel function included in (11),

$$
\begin{aligned}
\int_{0}^{\infty} \mathrm{d} \rho \int_{0}^{2 \pi} \mathrm{d} \varphi \rho \frac{2 J_{1}(k R \rho)}{k R \rho} & =\frac{4 \pi}{k^{2} R^{2}} \int_{0}^{\infty} \mathrm{d}(k R \rho) J_{1}(k R \rho) \\
& =\frac{4 \pi}{k^{2} R^{2}} .
\end{aligned}
$$

Using a simple relation,

$$
\int_{0}^{2 /(k R)} \mathrm{d} \rho \int_{0}^{2 \pi} \mathrm{d} \varphi \rho=\frac{4 \pi}{k^{2} R^{2}},
$$

we can replace the integral limit of $\rho$ by $2 /(k R)$ and $2 J_{1}(k R \rho) / k R \rho$ and also the exponential term in the integral by unity because the distribution $E_{\mathrm{obj}}$ contributes to the integral only when $\rho$ is almost zero as mentioned above. Finally, the distribution $E_{\mathrm{obj}}$ and phase term can be regarded as a constant $E_{\mathrm{obj}}\left(a \xi_{2} / b, a \eta_{2} / b\right)$ because the variations of them are slow in the $\rho$ range of $[0,2 /(k R)]$ which corresponds to the size of a few wavelengths on the object plane. Thus we acquire the relation,

$$
\begin{aligned}
E_{\mathrm{img}}\left(\xi_{2}, \eta_{2}\right) \approx & \frac{a}{4 \pi b} \exp \left(i k(b-a)-i k \frac{a}{b} \frac{\xi_{2}^{2}+\eta_{2}^{2}}{2 f}\right) \\
& \times \int_{0}^{2 /(k R)} \mathrm{d} \rho \int_{0}^{2 \pi} \mathrm{d} \varphi \rho E_{\mathrm{obj}}\left(\frac{a \xi_{2}}{b}, \frac{a \eta_{2}}{b}\right) \\
= & \frac{a}{b} E_{\mathrm{obj}}\left(\frac{a \xi_{2}}{b}, \frac{a \eta_{2}}{b}\right) \\
& \times \exp \left(i k(b-a)+i k \frac{a \xi_{2}^{2}+\eta_{2}^{2}}{2 f}\right) .
\end{aligned}
$$

We gather here the condition and relations we seek,

$$
\begin{gathered}
\frac{1}{f}=-\frac{1}{a}+\frac{1}{b}, \\
E_{\text {img }}\left(\xi_{2}, \eta_{2}\right)=\frac{a}{b} E_{\text {obj }}\left(\frac{a \xi_{2}}{b}, \frac{a \eta_{2}}{b}\right) \\
\times \exp \left(i k(b-a)-i k \frac{a}{b} \frac{\xi_{2}^{2}+\eta_{2}^{2}}{2 f}\right), \\
\frac{\xi_{1}}{a}=\frac{\xi_{2}}{b}, \frac{\eta_{1}}{a}=\frac{\eta_{2}}{b} .
\end{gathered}
$$

Since these apply for any signs of $a, b$, and $f$, (16) and (17) holds whenever $a$ and $b$ meet the lens formula (15). We can clearly and concisely derive (15) to (17) which can be seen in [7].

\section{RELATION TO GEOMETRICAL OPTICS}

When we have a closer look at (16) and (17), there is a one-to-one correspondence between the points $\left(\xi_{1}, \eta_{1}\right)=$ $\left(a \xi_{2} / b, a \eta_{2} / b\right)$ on the object plane and $\left(\xi_{2}, \eta_{2}\right)$ on the image plane. It means that we can treat the field distribution with the geometrical optics on the planes where the lens formula holds. Whenever an imaging system having an object and its image with no aberrations is formed by the geometrical optics, (16) can be applied to it. In addition, the geometrical optics allows us to know an equivalent single lens system obtained by a ray tracing simulation or by the formula combining two focal lengths of $f_{1}, f_{2}$,

$$
\frac{1}{f_{\mathrm{comb}}}=\frac{1}{f_{1}}+\frac{1}{f_{2}}-\frac{d}{f_{1} f_{2}},
$$

where $f_{\text {comb }}$ is the equivalent focal length and $d$ is the distance between the two lens. It is important to consider an equivalent single lens system because there are difficulties in applying the relations as many times as the number of optical elements in a system considered, and because the relations cannot be applied to one of a pair of elements which works as one lens. Using a ray tracing simulation, we obtain an equivalent focal length, and then, can apply the condition and relations derived here to complicated systems with many focusing elements, or with each element not having a traditional focal length, e.g., a mirror with a free-form surface. Thus, (16) can be applied to an arbitrary optical system because an equivalent single lens system of it is obtained by the geometrical optics.

Next, we would like to consider how to apply (16) to a wide FOV system. In general, various beams each propagate on separate paths in a wide FOV system but coincide at "pupils", where all of them are piled up on the same position. The pupil is a fundamental concept in the geometrical optics [9]. The lens formula always holds on all of the pupils because they are images of one another. In addition, it is most important that beam patterns are defined at either pupil; for the entrance pupil they are far-field patterns, for the exit pupil they are "receiver" patterns, i.e. point-spread functions. When we set an electric field distribution on a specific pupil and apply (16) to it and the entrance (exit) pupil, the electric field distributions of all the beams are the same one unrelated to propagating directions and always independent of frequencies on them. 
Thus, a frequency-independent system is simply obtained. All we have to do is to investigate positions of pupils with a ray tracing simulation or (18), and to determine beam parameters on one of the pupils.

\section{VERIFICATION OF THE RELATION}

In this section, we verify the relation (16) with some examples. Firstly, we apply (16) to the Gaussian beam and derive the conditions of [6]. Assuming an on-axis Gaussian beam with a beam waist of $w_{0}$ which is located at $a_{0}$,

$$
\begin{aligned}
E_{\mathrm{obj}}\left(x_{1}, y_{1} ; a\right)= & \sqrt{\frac{2}{\pi w(a)^{2}}} \exp \left[-\frac{x_{1}{ }^{2}+y_{1}^{2}}{w(a)^{2}}+i k\left(a-a_{0}\right)\right. \\
& \left.-i k \frac{x_{1}^{2}+y_{1}^{2}}{2 R(a)^{2}}-i k \varphi(a)\right] \\
w(a)= & w_{0} \sqrt{1+\left(\frac{2\left(a-a_{0}\right)}{k w_{0}^{2}}\right)^{2}}, \\
R(a)= & -\left(a-a_{0}\right)\left[1+\left(\frac{k w_{0}^{2}}{2\left(a-a_{0}\right)}\right)^{2}\right] \\
\varphi(a)= & -\tan ^{-1} \frac{2\left(a-a_{0}\right)}{k w_{0}^{2}},
\end{aligned}
$$

we substitute these equations in (16), and obtain the electric field after it passes through a focusing element,

$$
\begin{aligned}
& E_{\text {img }}\left(x_{2}, y_{2} ; b\right)= \frac{a}{b} E_{\text {obj }}\left(\frac{a x_{2}}{b}, \frac{a y_{2}}{b} ; a\right) \\
& \times \exp \left(i k(b-a)-i k \frac{a}{b} \frac{x_{2}{ }^{2}+y_{2}{ }^{2}}{2 f}\right) \\
&=\operatorname{sgn}\left(\frac{a}{b}\right) \sqrt{\frac{2 a^{2}}{\pi b^{2} w(a)^{2}}} \exp \left[-\frac{x_{2}{ }^{2}+y_{2}^{2}}{b^{2} w(a)^{2} / a^{2}}+i k\left(b-b_{0}\right)\right] \\
& \\
& \times \exp \left[-i k \frac{x_{2}^{2}+y_{2}^{2}}{2}\left(\frac{a^{2}}{b^{2} R(a)}+\frac{a}{b f}\right)\right] \\
& \times \exp \left[-i k\left(\varphi(a)+a_{0}-b_{0}\right)\right],
\end{aligned}
$$

where $b=(1 / f+1 / a)^{-1}, b_{0}$ is the position of the beam waist after the focusing element. We here replace a few terms,

$$
\begin{aligned}
w^{\prime}(b)^{2} & =\frac{b^{2} w(a)^{2}}{a^{2}}, \\
\frac{1}{R^{\prime}(b)} & =\frac{a^{2}}{b^{2} R(a)}+\frac{a}{b f}, \\
\varphi^{\prime}(b) & =\varphi(a)+a_{0}-b_{0} .
\end{aligned}
$$

Thus we arrange them using the lens formula and acquire the conditions shown in [6],

$$
\begin{aligned}
\frac{w^{\prime}(b)^{2}}{b^{2}} & =\frac{w(a)^{2}}{a^{2}}, \\
b\left(1+\frac{b}{R^{\prime}(b)}\right) & =a\left(1+\frac{a}{R(a)}\right) .
\end{aligned}
$$

Next, we apply to a one spherical mirror system with a focal length $f=150 \mathrm{~mm}$. We run a Physical Optics (PO) simulation using GRASP [10] and compare simulation results with the expected distributions by (16). Three Gaussian beams enter the mirror; a $500 \mathrm{GHz}$ on-axis beam, a $500 \mathrm{GHz}$ beam $10 \mathrm{~mm}$ off the axis (Fig. 2), and a $1 \mathrm{THz}$ beam with the same beam

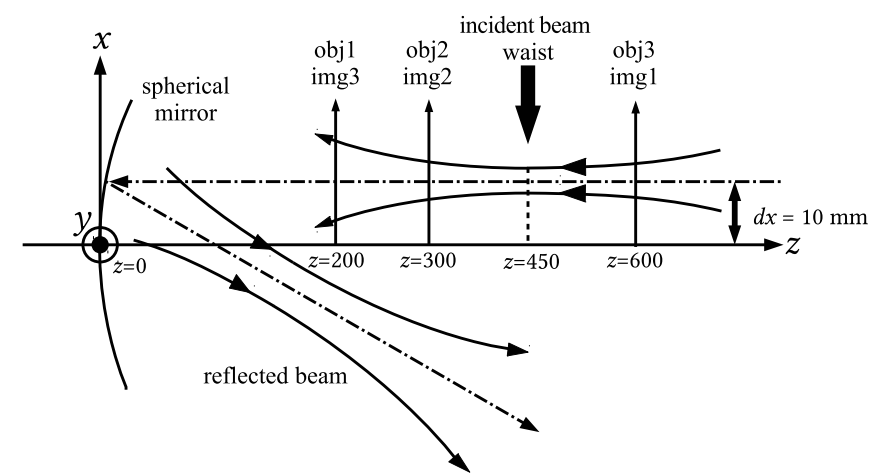

Fig. 2. One mirror simulation model for a $500 \mathrm{GHz}$ off-axis beam. An incident beam propagates $10 \mathrm{~mm}$ off the axis and in parallel to it, and a reflected beam does not in parallel to it as shown. Both beams are in the region $z>0$. The planes (obj1, img1), (obj2, img2), and (obj3, img3) are pairs of images that satisfy the lens equation.

radius and radius of curvature of a wavefront at the plane obj2 as those of the $500 \mathrm{GHz}$ off-axis beam. The beam waists of the $500 \mathrm{GHz}$ beams are located at $450 \mathrm{~mm}$. We first investigate the field distribution of the incident beams on the planes obj1, obj2, and obj3 at $z=200,300$, and $600 \mathrm{~mm}$, respectively. The beams reflect and then we calculate the distribution of the reflected beams on the planes img1, img2, and img 3 at $z=600,300$, and $200 \mathrm{~mm}$, respectively, by two ways, PO simulation and substituting the distribution on the planes obj1, obj2, and obj3, for (16). Finally we compare the distribution by PO simulation and (16).

Fig. 3 and Fig. 4 show results of the $500 \mathrm{GHz}$ beams. In Fig. 3 , the electric distributions expected by (16) agree with those calculated by PO very well. The finite size of the spherical mirror affects the distribution distant from the center as shown in Fig. 3 (b), (c), (e), and (f). The distribution at the img1 (Fig. 3 (a) and (d)) should also be affected in a region $|x|>$ $50 \mathrm{~mm}$, where we have not calculated with PO. An effect of mirror aberrations which fills in nulls between sidelobes is also seen. The results of calculating the phase by PO and (16) are shown in Fig. 4. The results of (16) also agree with that by PO near the center where there is not an effect of the mirror size. We confirm that (16) holds at the position satisfying the lens formula with the $500 \mathrm{GHz}$ beams.

We finally confirm whether the field distributions and phase on the specific positions are independent of the beam frequencies. The $500 \mathrm{GHz}$ and $1 \mathrm{THz}$ off-axis beams are used. The results are shown in Fig. 5. Except for the region where the effect of the finite mirror size exists, the distribution and wavefront of both frequencies shows the same shape at the plane img2. This confirms that the condition for fields independent of frequency is that the plane positions which are taken into consideration satisfy the lens formula, $1 / f=-1 / a+1 / b$, and that the distribution and phase have the relations expressed by (16).

\section{Applying to Three Mirror Telescope}

We apply (16) to a three-mirror telescope in Fig. 6. The primary mirror is an off-axis paraboloid with a diameter of $300 \mathrm{~mm}$. The secondary mirror is an off-axis ellipsoid. The 


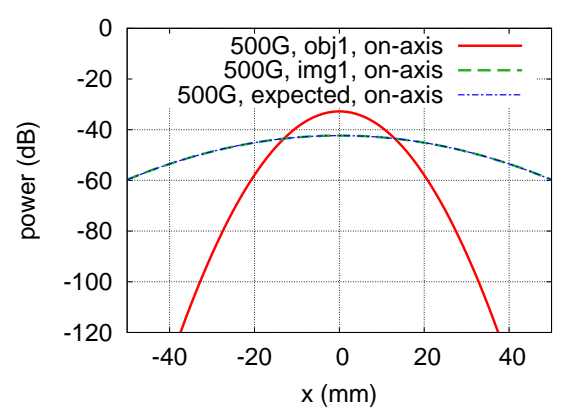

(a) $500 \mathrm{GHz}$ on-axis beam on the obj1 and img1.

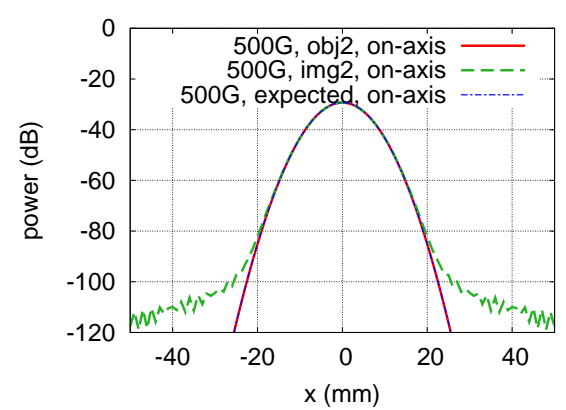

(b) $500 \mathrm{GHz}$ on-axis beam on the obj2 and img2.

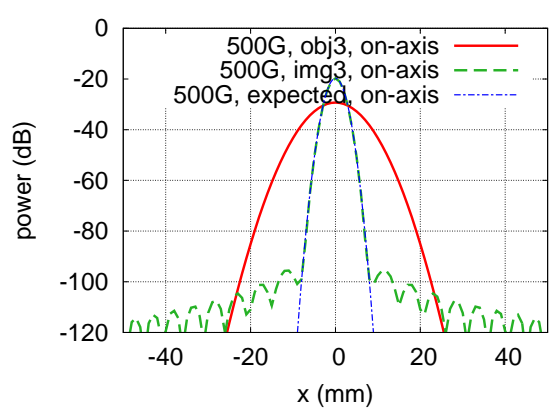

(c) $500 \mathrm{GHz}$ on-axis beam on the obj3 and img3.
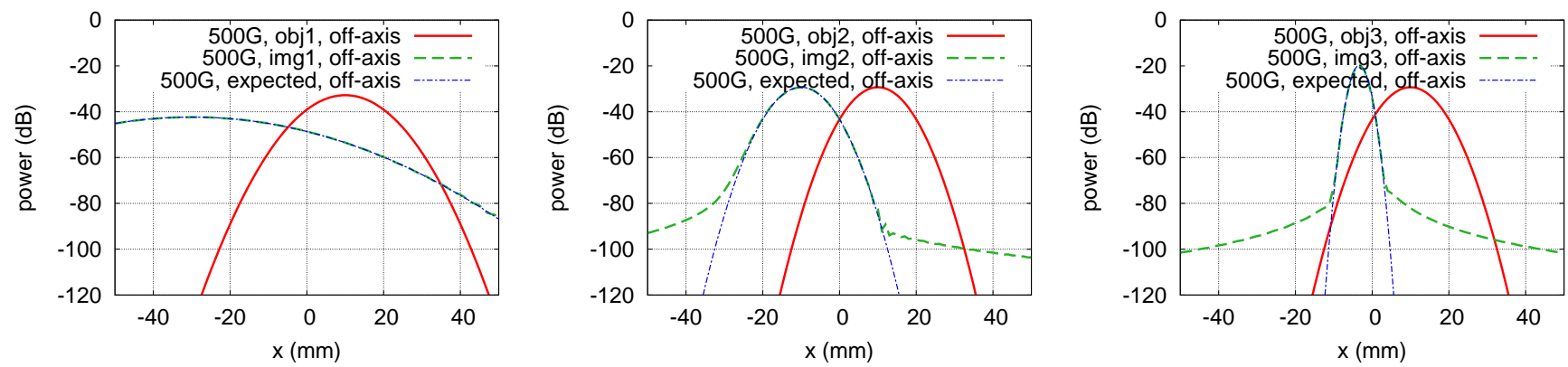

(d) $500 \mathrm{GHz}$ off-axis beam on the obj1 and img1. (e) $500 \mathrm{GHz}$ off-axis beam on the obj2 and img2. (f) $500 \mathrm{GHz}$ off-axis beam on the obj3 and img3.

Fig. 3. Calculated electric fields on the obj and img planes. The red (solid) line is the incident beam, the green (dashed) line the reflected beam by PO. The blue (dashed-dotted) line is the expected distribution by (8) from the incident beam distribution (the red-solid line).

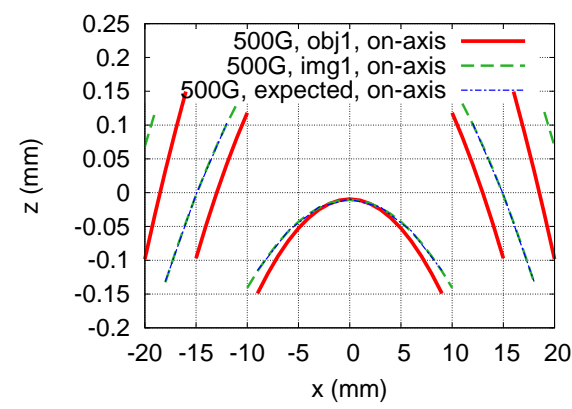

(a) $500 \mathrm{GHz}$ on-axis beam on the obj1 and img1.

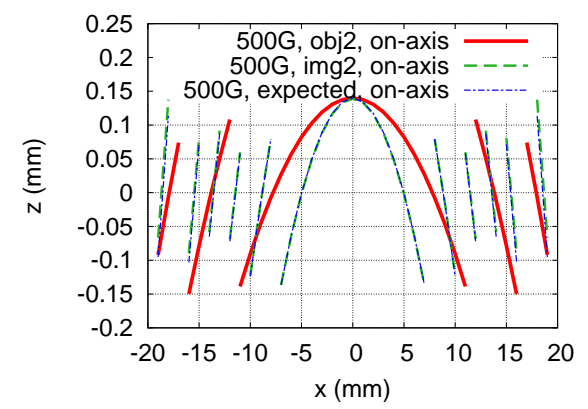

(b) $500 \mathrm{GHz}$ on-axis beam on the obj2 and img2.

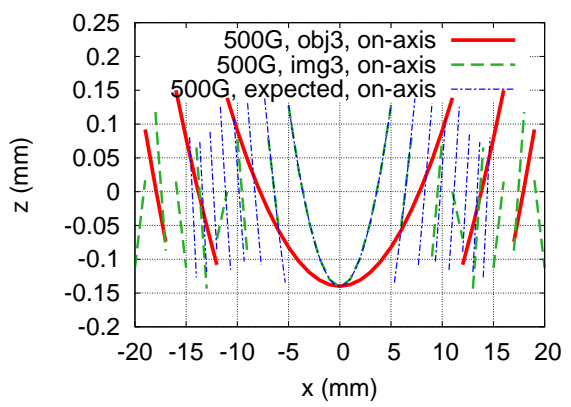

(c) $500 \mathrm{GHz}$ on-axis beam on the obj3 and img 3 .

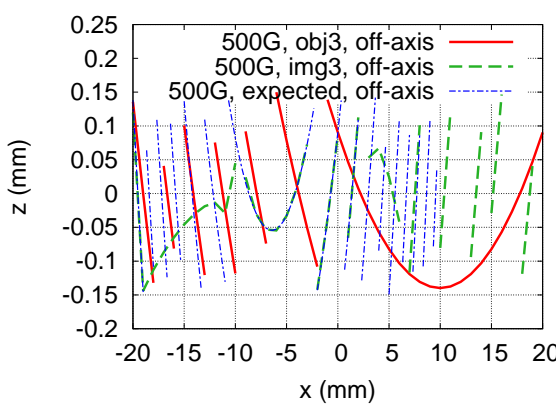

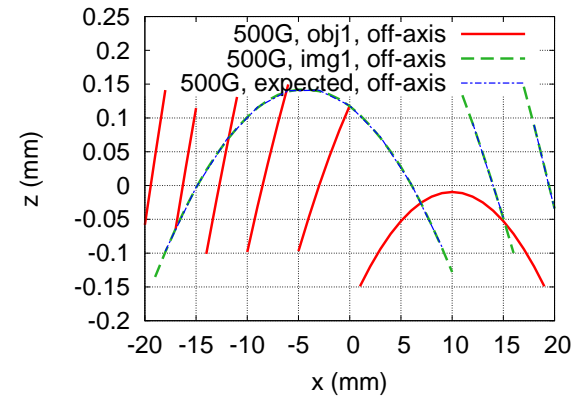

(d) $500 \mathrm{GHz}$ off-axis beam on the obj1 and img1.

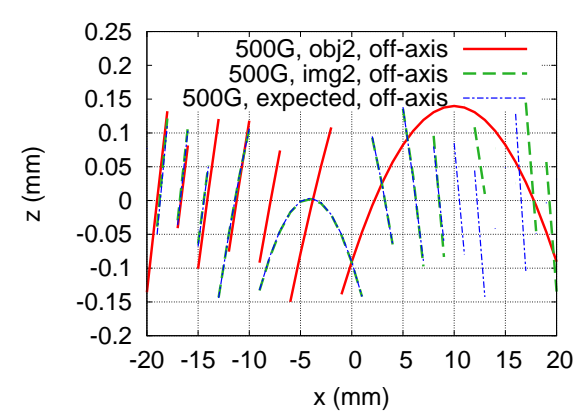

(e) $500 \mathrm{GHz}$ off-axis beam on the obj2 and img2

. (f) $500 \mathrm{GHz}$ off-axis beam on the obj3 and img3.

Fig. 4. Cross sections of equiphase surfaces (wavefront) on the plane $y=0$ at the obj and img planes. Figures (a) to (f) correspond to Fig. 3 (a) to (f), respectively. The red (solid) line is the incident beam, the green (dashed) line the reflected beam by PO. The blue (dashed-dotted) line is expected by (16) from the incident beam distribution (the red-solid line). 


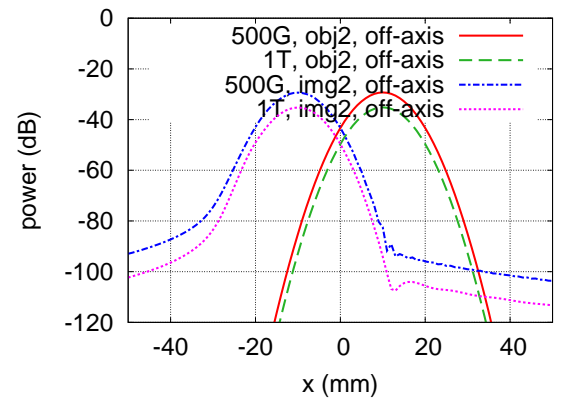

(a)

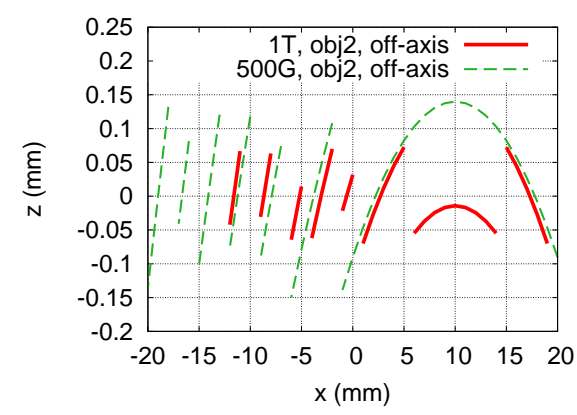

(b)

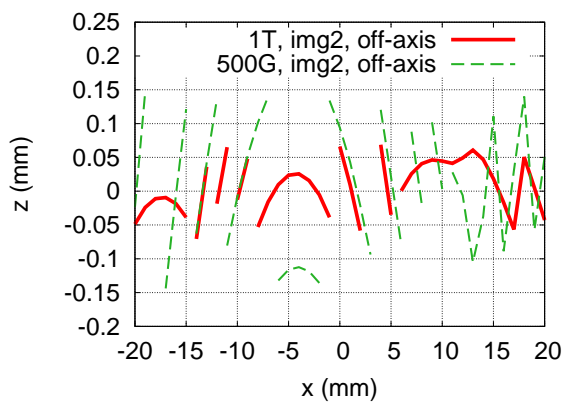

(c)

Fig. 5. The distribution and equiphase surface (wavefront) on the obj2 and img2. (a) The red (solid) line and green (dashed) line represent the distribution of the $500 \mathrm{GHz}$ and $1 \mathrm{THz}$ off-axis incident beam, respectively. They are similar one. The blue (dashed-dotted) line and magenta (dotted) line is $500 \mathrm{GHz}$ and $1 \mathrm{THz}$ off-axis reflected beam distribution, respectively. (b) The red (solid) line and green (dashed) line represent cross sections of the equiphase surfaces of the $500 \mathrm{GHz}$ and $1 \mathrm{THz}$ off-axis incident beams, respectively. (c) The red (solid) line and green (dashed) line represent cross sections of the equiphase surfaces of the $500 \mathrm{GHz}$ and $1 \mathrm{THz}$ off-axis reflected beam, respectively. They have the same shape.

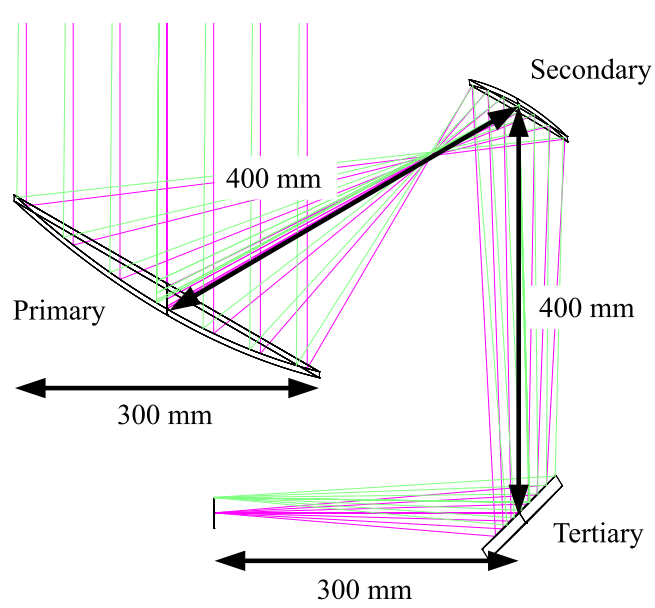

Fig. 6. Layout of three mirrors. The primary and secondary mirror fold beams by 60 degrees, and the tertiary folds by 90 degrees.

tertiary mirror is an off-axis hyperboloid. The parameters of the three mirrors are determined following [11]. We use a ray tracing program, Zemax [12]. The telescope has a focal length of about $1663 \mathrm{~mm}$. This telescope has a wide FOV of 1 degree at $1 \mathrm{THz}$ without vignetting in which there are scarecely aberrations. The limiting aperture at the secondary defines a stop; its images shown in Fig. 7 are the entrance and exit pupils, on which the lens formula holds allowing us to apply relation (16). In this case, an equivalent $a$ and $b$ are about $-4236 \mathrm{~mm}$ and $2731 \mathrm{~mm}$, respectively, at which the pupils are located.

We carry out calculations with PO. Four beams pass through the telescope from the focal plane. One of them is a $500 \mathrm{GHz}$ on-axis beam, the second is a $500 \mathrm{GHz} 0.5$ degrees off-axis apart from the center of the FOV, the others are the same beams as the $500 \mathrm{GHz}$ ones, except for the frequency of $1 \mathrm{THz}$. The off-axis beams propagate toward the center of the exit pupil (Fig. 7). An edge taper is set to be $-15 \mathrm{~dB}$ at the secondary. Combining this taper with the radius of stop on the secondary gives a beam radius $w_{0}$ to be used in (16). A radius of curvature of wavefront is estimated from a distance between

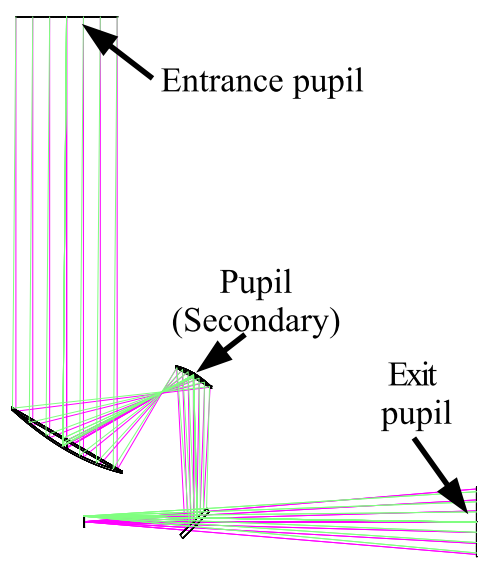

Fig. 7. Three pupils of the considered telescope. One of them is located at the secondary. The Entrance pupil is an image of the secondary by the primary, and the exit pupil is by the tertiary. The magenta beam is on the axis of the telescope. The green beam is 0.5 degrees off the center of the FOV.

the focal plane and the exit pupil. With these parameters, if the field distribution at the exit pupil is independent of the frequencies, we can expect that the distribution on the entrance pupil will be also independent of the frequencies.

Fig. 8 shows the distributions on the exit and entrance pupil of the telescope. The $500 \mathrm{GHz}$ and $1 \mathrm{THz}$ distributions are similar on the exit pupil and keep their own shape on the entrance pupil. The fine dotted lines in Fig. 8 represent the expected distributions by (16). It is shown that the field distributions on the entrance pupil do not depend on the beam frequencies and specific information of the telescope, e.g. the number of mirrors, focal lengths of each, and so on. Although (16) frees us from having to know details of the system, it does not tell whether the actual apertures of mirrors are sufficient to transmit beams without significant distortion; these aperture sizes must be determined by other methods, e.g., PO simulation. It is also unknown whether efficiencies of a whole system are high or not. Efficiencies like coupling a image and detectors have frequency-dependence. Other evaluations of efficiencies are needed. 


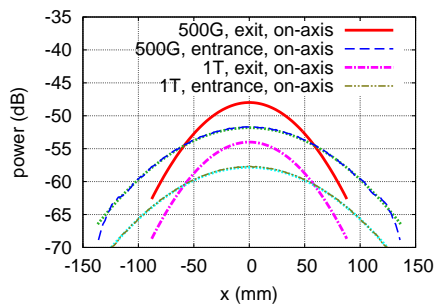

(a) On-axis beams, field along $x$ axis.

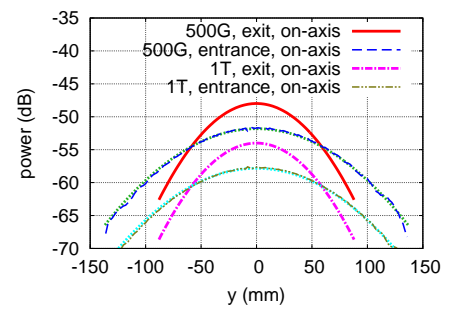

(b) On-axis beams, field along $y$ axis.

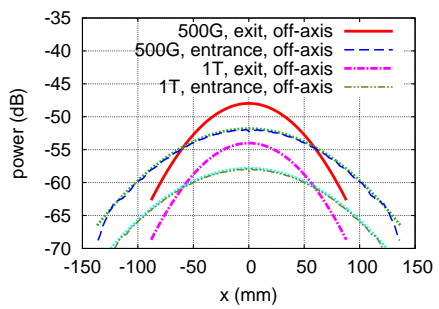

(c) Off-axis beams, field along $x$ axis.

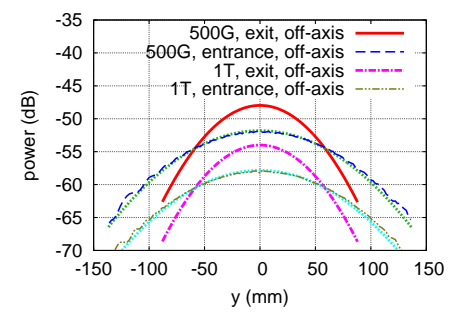

(d) Off-axis beams, field along $y$ axis.

Fig. 8. Three-mirror telescope: field distributions on the entrance and exit pupil. The red (solid) line is the distribution of the $500 \mathrm{GHz}$ beam at the exit pupil, the blue (dashed) line is that at the entrance pupil. The magenta (dashed-dotted) and greenish brown (dashed-dotted-dotted) represents the distribution of the $1 \mathrm{THz}$ beam at the entrance and exit pupil, respectively. The cyan and green (fine dotted) lines are expected from the distribution on the exit pupil.

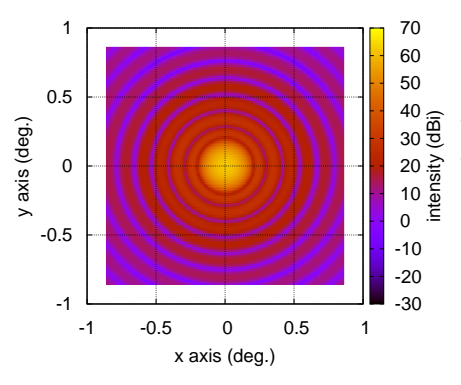

(a) $500 \mathrm{GHz}$ on-axis beam.

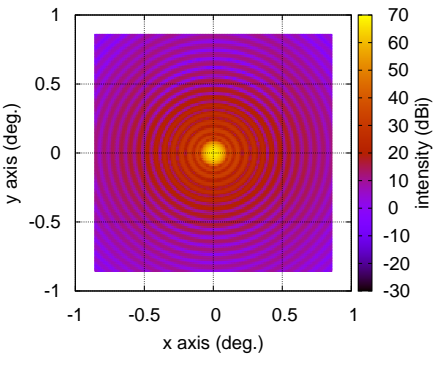

(b) $1 \mathrm{THz}$ on-axis beam.

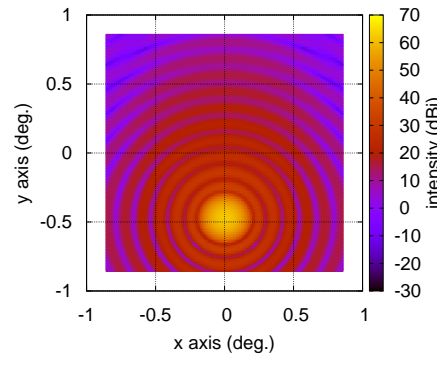

(c) $500 \mathrm{GHz}$ off-axis beam.

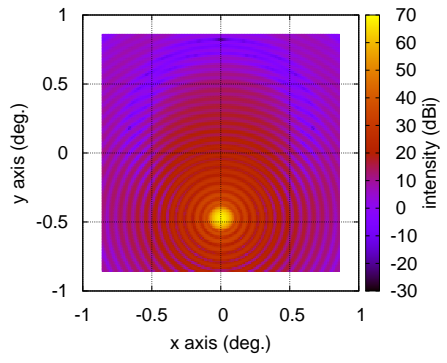

(d) $1 \mathrm{THz}$ off-axis beam.

Fig. 9. Beam patterns of the telescope.

We also confirm beam patterns of this telescope. Fig. 9 shows beam patterns of the four beams on the far-field sphere. They are axially symmetric and have beam sizes expected from the diameter of the entrance pupil.

\section{CONCLUSION}

Starting from the Fresnel diffraction integral, we derive a condition for wide-FOV systems to form images independent of frequency. The condition can be expressed simply using the well-known lens formula. We can obtain images independent of frequency where and only where the lens formula holds. The relation of the field distributions between the object and image planes is acquired. The relation corresponds completely to the geometrical optics. The relation of the phase is directly derived in this paper, which has never been mentioned to the best of our knowledge. Numerical simulations verify the relations with a simple model consisting of one mirror, and confirm that we can apply them to a wide FOV telescope with a model of a three-mirror telescope. To design a wide FOV telescope, all we have to do is to calculate the equivalent focal length to an optical system considered and the position of the entrance and exit pupil with the geometrical optics or ray tracing software, and to determine beam parameters at the pupils.

\section{ACKNOWLEDGMENT}

The authors would like to thank the anonymous referees for their comments improving the paper. The authors are grateful to the National Institute of Information and Communications
Technology (NICT) for supporting a PO simulation. H. I. is financially supported by Grant-in-Aid for Japan Society for the Promotion of Science (JSPS) Fellows.

\section{REFERENCES}

[1] J. E. Carlstrom, P. A. R. Ade, K. A. Aird et al., "The 10 meter south pole telescope," Publications of the Astronomical Society of the Pacific, vol. 123, no. 903, pp. 568-581, 2011.

[2] J. W. Fowler, M. D. Niemack, S. R. Dicker et al., "Optical design of the Atacama Cosmology Telescope and the Millimeter Bolometric Array Camera," Appl. Optics, vol. 46, no. 17, pp. 3444-3454, 2007.

[3] S. Padin, M. Hollister, S. Radford et al., "CCAT Optics," Proc. SPIE, vol. 7733, 77334Y, 2010.

[4] V. Y. Terebizh, "A Purely Reflective Large Wide-Field Telescope," Bulletin of the Crimean Astrophysical Observatory, vol. 104, pp. 179186, 2008.

[5] H. Kataza, T. Wada, I. Sakon, N. Kobayashi, Y. Sarugaku, N. Fujishiro, et al., "Mid-infrared Camera and Spectrometer on board SPICA," Proc. SPIE, vol. 8442, 84420Q, 2012.

[6] T. Chu, "An Imaging Beam Waveguide Feed," IEEE Trans. Antennas Propag., vol. 31, no. 4, pp. 614-619, 1983.

[7] D. H. Martin and J. W. Bowen, "Long-Wave Optics," IEEE Trans. Microw. Theory and Tech., vol. 41, no. 10, pp. 1676-1690, 1983.

[8] J. W. Goodman, "Wave-Optics Analysis of Coherent Optical Systems," in Introduction to Fourier Optics, 2nd ed. New York: McGraw-Hill, 1968.

[9] M. Born and E. Wolf, "Geometrical Theory of Optical Imaging," in Principles of Optics, 6th ed. Cambridge, United Kingdom: Cambridge University Press, pp. 186-187, 1999

[10] TICRA Engineering Consultants, "GRASP8: general reflector and antenna farm analysis program package," Copenhagen, Denmark.

[11] S. Chang, "Elimination of linear astigmatism in off-axis three-mirror telescope and its applications," Proc. SPIE, vol. 8860, 88600U, 2013.

[12] Radiant Zemax, LLC, "Zemax 13 EE: Optical Design Program”, Redmond. 